\title{
Sensitivity Analysis of Simplified Low Cost Converging Thermal Wave Technique for Thin Bilayer Solid
}

\author{
M. S. Husin*, M. M. Moksin*, S. Jibrin*, B. Z. Azmi*, W. M. M. Yunus*, M. Waziri** \\ * Department of Physics, Faculty of Science, Universiti Putra Malaysia, Malaysia \\ ** Department of Mathematics, Faculty of Science, Universiti Putra Malaysia, Malaysia
}

\begin{tabular}{l} 
Article Info \\
Article history: \\
Received Jun 15, 2014 \\
Revised Aug 12, 2014 \\
Accepted Aug 22, 2014 \\
\hline Keyword: \\
Camera flash \\
Simplified converging thermal \\
wave technique \\
Thermal diffusivity \\
Thin bilayer
\end{tabular}

Article Info

Article history:

Received Jun 15, 2014

Revised Aug 12, 2014

Accepted Aug 22, 2014

Thin bilayer

\begin{abstract}
In this paper, the sensitivity analysis of thermo physical parameters of the semi-infinite bilayer was presented by using a simplified converging thermal wave model. This is done under the consideration that radial flow of converging thermal wave substantially dominates in the first layer and axial thermal wave, dominates in the second layer. The sensitivity of the correlated parameters due to the $5 \%, 10 \%$ and $100 \%$ of increment were evaluated.. The results of the calculated temperature were simulated by Mathematica software, in order to generate a sensitivity analysis and examined by graphs. From this analysis, we concluded the optimum conditions, hence to be applied in the real experiment. Our merit on this report is for introducing a simple method of optical transient heating by using low cost equipment such as camera's flash lamp and thermocouple, which may also apply to the short pulse laser measurement. A brief theory of the present work is presented and the results obtained from the simulation are discussed.
\end{abstract}

Copyright () 2014 Institute of Advanced Engineering and Science. All rights reserved.

\section{Corresponding Author:}

\section{S. Husin,}

Department of Physics, Faculty of Science,

Universiti Putra Malaysia,

Serdang, Selangor, Malaysia.

Email:

\section{INTRODUCTION}

The growing demand on miniatured size of electronic devices raised the impact of challenge in measuring thermo-physical quantities and developing new techniques suitable for low dimensional applications in particular for thin layers and films. Characterizing thermo physical properties including thermal diffusivity and thermal conductivity of the decreasing size dependent materials are urgently needed as they are rigidly no longer inherited its bulk properties. Among the two properties thermal diffusivity is simpler to measure which is then used to determine thermal conductivity of the materials by knowing their specific heat capacity and density.

\subsection{Flash Technique}

Thermal diffusivity measurement by using flash method as introduced by Parker et al. [1] is widely accepted in industries and research institutes since early 60's. It consists of an optical irradiating front surface of the sample for a duration significantly smaller compared with the transit time of the induced thermal waves through the sample. The sample is normally in the form of a thin disc and the temperature history is monitored on its rear face. The thermal diffusivity is determined from the half-rise time $t_{1 / 2}$ of the rear face temperature. This technique is designed to allow only one dimensional heat flow in order to simplify the solution of the heat diffusion equation.

Since its inception in 1961, the flash method has been accepted as a standard technique for measuring thermal diffusivity of solids. Unfortunately, this method has been unsuccessful in the case of 
highly conducting thin solids when the diffusion time is less than the duration of optical radiation. The flash method requires fairly simple specimen, furnaces, and associated control, but it is unsuccessful to measure thermal diffusivity of ultra thin sample especially for high diffusivity materials without the presence of ultrashort pulsed laser. Kehoe et al. [2] have successfully measured the thermal diffusivity of high conducting metal foils with the thickness approximately approaching $30 \mu \mathrm{m}$ using the same method proposed by Parker by using nanoseconds pulsed laser. Any application on thinner or higher thermal diffusivity samples would require faster laser that would easily causes laser damage to the samples besides requiring a much faster detector to monitor the rear temperature

\subsection{Converging Thermal Wave Technique}

Converging thermal wave technique was proposed by P. Cielo et al [3] to overcome the problems associated with thin samples. They used an annular beam heating rather than a beam spot as used by Parker et al., by inserting an axicon lens placed in between the sample front surface and the sample. The locally induced heat along the annular would partly propagate inward and would be detected at the centre of the annulus. This means the measurement involves with in-plane heat diffusion, which allow longer diffusion time, in contrast with axial heat diffusion in Parker's method. This scheme also allows stronger signal to be detected as it was a collection of energy that converges to the centre of the rear surface of the specimen.

The converging thermal wave technique was found most suitable for the sufficiently thin sample [4][5] such that in-plane heat mostly dominates the heat diffusion process. In this method, it is pertinent to have an optical heating beam in the shape of a very thin ring to reduce the error in the annulus's radius measurement. This requires a highly focused laser source that could exceed the damage threshold of the sample. Otherwise a signal amplification and data averaging over up to thousand signals are standard procedure which may allow larger annulus radius to be used to mitigate the error in the measurement. This may not possible for infinitely thin, high thermal conductivity and light-reflect and semi transparent materials (such as metals), which only absorbed very small portion of light, causing poor signal for detection unless highly light absorbing coat is applied on the front surface. However, introducing another layer of highly absorbing material on the top of the surface leads to new issue when its thickness becomes comparable to the specimen. The sample becomes a bilayer and the technique would measure the effective thermal diffusivity of the bilayer. By that, the new converging thermal wave technique must be considered to include this effect.

In this paper, we present the sensitivity analysis of thin bilayer sample using simplified converging thermal wave technique for the implementation using a low cost setup by using camera flash (in order to generate thermal wave signals in the specimen) by radiating the front surface of the opaque layer and the rear surface temperature is monitored using an ordinary thermocouple.

\section{THEORETICAL}

\subsection{Single Layer Converging Thermal Wave}

Let $T(r, t)$ represents the temperature of a semi infinite specimen at a point $\mathrm{r}$, at time $\mathrm{t}$. As the specimen is infinitely large in radius, $r$ as compared to the thickness $z$, the ideal case of a two dimensional case can be applied to solve the heat equation problem.

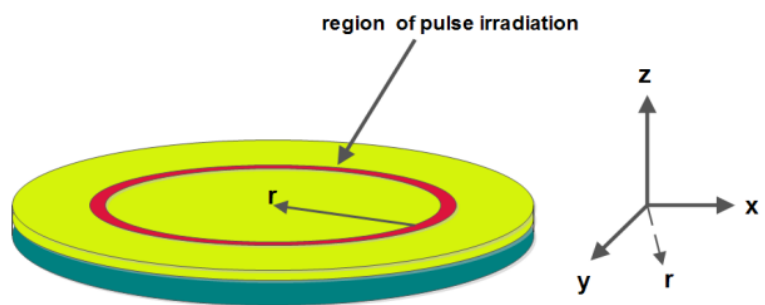

Figure 1. Schematic diagram of semi infinite bilayer heated by a ring pulsed irradiation

A semi-infinite of bilayer model is presented and employed in the converging thermal wave configuration. The thermal conductivities of the first and second layer are $\mathrm{k} 1$ and $\mathrm{k} 2$, respectively. Thickness of the first layer, $\mathrm{d}$ and second layer extends from $\mathrm{z}=\mathrm{d}$ to infinity. A surface of homogeneous specimen instantaneously irradiates on a 'ring' region by a concentric heat pulse with energy $\mathrm{Q}(\mathrm{W} / \mathrm{m} 2)$ at time $\mathrm{t}=0$ and $\mathrm{T}(\mathrm{r}, \mathrm{t})=\mathrm{T} 1$ ( not exceed $10 \mathrm{~K}$, which allows the effect of heat losses are small and negligible). The thermal wave generated on the surface, then diffuses to the center by radius $r$. Assuming the entire specimen is 
uniformly homogenous with constant thermal properties, the heat conduction equation for this problem can be written as:

$$
\frac{\partial T}{r^{2} \partial t}=\alpha \frac{\partial T}{\partial r}\left(r^{2} \frac{\partial T}{\partial r}\right)
$$

The temperature $\mathrm{T}(\mathrm{t})$ that satisfying the heat conduction equation for the semi infinite disc, $\mathrm{T}=\mathrm{T} 0$ $=0 \mathrm{~K}$, at $\mathrm{t}=0 \mathrm{~s}$ (at equilibrium state) is given by [5]:

$$
T(r, t)=\frac{Q}{4 \pi \alpha t^{p}} \exp \left[-\frac{r^{2}+d^{2}}{4 \pi \alpha t}\right]
$$

Equation (2) can be simplified by normalization approach [6] by dividing Equation (2) with the maximum value of $\operatorname{Tmax}(r, t m)$, therefore $\mathrm{Q} /(4 \pi \alpha)$ vanished and Equation (2) becomes simplified with the maximum amplitude achieving 1 (dimensionless) and only dependent on the radius ( $r$ ) and thermal diffusivity $(\alpha)$ which is written in Equation (3). The constant $p$ in Equation (2) is related to the dimension number applied to the samples. For example the value of $\mathrm{p}$ in two dimensional is 1 (2 divided by 2 ) where the thickness of the specimen, $\mathrm{d}$ can be neglected when it comes infinitely small compare to the length of radius r. For the three dimensional case, the value of $\mathrm{p}$ becomes 1.5 (3 divided by 2 ).

$$
\frac{T(t)}{T_{\max }\left(t_{m}\right)}=\frac{t_{m}}{t} \exp \left[\frac{r^{2}}{4 \alpha}\left(\frac{1}{t_{m}}-\frac{1}{t}\right)\right]
$$

If Equation (2) is differentiated, the peak time at which the amplitude reaches a maximum, tm, can be used to calculate the initial value of thermal diffusivity by equation (4), then to be employed in the curve fitting evaluation efficiently and accurate. After differentiate, Equation (2) becomes [7]:

$$
\alpha=\frac{r^{2}}{4 p t_{m}}
$$

\section{SIMPLIFIED BILAYER CONVERGING THERMAL WAVE}

In two layer structure, we have adopted an inverse scattering algorithm applied to infrared thermal wave images developed by Favro et al. [8]-[9]. Favro's equation is related in detecting defects in the anisotropic monolayer sample irradiated by a flash lamp due to axial thermal wave scattering and reflection parameters. We take Favro's model in our approach to construct theoretical model of two layer structure since the thermal wave has reflected and reabsorbed repeatedly at the interface can be adopted. Without defect scattering, and by considering first layer being infinitely thin compared to the second layer where the radial converging thermal wave is dominant (which can be achieved by using thin metallic layer or any high diffusivity materials), and axial thermal wave is dominant in the second layer (thicker substrate and lower thermal diffusivity than the first layer) as shown in Figure 2, converging thermal wave term [4] has been substituted into an axial equation [8] as in the following form:



Where,

$$
\mathrm{A}_{\mathrm{m}}=\sqrt{\frac{r^{2}}{\mathrm{a} 2}+\frac{(2 m-1)^{2} l^{2}}{\mathrm{a} 2}}
$$

And,

$$
\begin{aligned}
& R=(1-\varepsilon) /(1+\varepsilon) \\
& \varepsilon=\frac{\mathrm{k} 2}{\sqrt{\mathrm{a} 2}} / \frac{\mathrm{k} 1}{\sqrt{\mathrm{a} 1}}
\end{aligned}
$$

Where:

$\mathrm{a} 1=$ thermal diffusivity of layer $1(\mathrm{~m} 2 \mathrm{~s}-1)$, 
$\mathrm{a} 2=$ thermal diffusivity of layer $2(\mathrm{~m} 2 \mathrm{~s}-1)$,

$\mathrm{r}=$ radius between ring and detection point $(\mathrm{m})$,

$\mathrm{R}=$ reflection coefficient,

$\mathrm{d}=$ thickness of layer $1(\mathrm{~m})$,

$l=$ thickness of layer $2(\mathrm{~m})$,

$\mathrm{Q}=$ energy absorbed by front surface radiation $(\mathrm{W} / \mathrm{m} 2)$,

$\mathrm{k} 1=$ thermal conductivity of layer $1(\mathrm{~W} / \mathrm{mK})$ and

$\mathrm{k} 2=$ thermal conductivity of layer $2(\mathrm{~W} / \mathrm{mK})$.

Converging thermal wave part is substituted in Equation (5) in the last exponential term taking from the relation in Equation (2). Parameter $r^{\prime}$ is referring to the ring's width. We have set that the value of $r^{\prime}$ is equal or comparable to the thickness (d) of the front surface layer 1 to obtain an efficient ratio between the thickness and heating area.

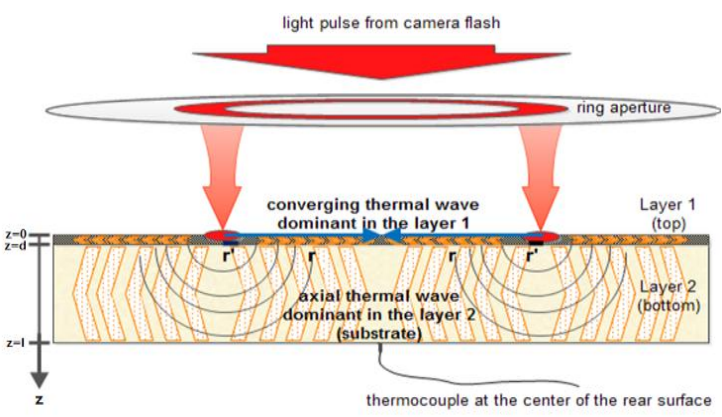

Figure 2. Schematic diagram of two layer model used in this work

\section{SENSITIVITYANALYSIS AND DISCUSSION}

In order to carry out the sensitivity analysis, due to non-homogeneous in magnitude and dimension of the parameter, we intended to analyze it by comparing the temperature changes by 5\%, $10 \%$ and $100 \%$ increment in each parameter once at a time with respect to the initial values (Table 1). The initial values been selected from the typical data used in practical, recruited for the reference as plotted in Figure 3. Three series increment by $5 \%, 10 \%$ and $100 \%$ for each parameter are shown in the Table 1 with constant iteration number $\mathrm{m}=2$, were chosen in the sensitivity analysis.

Table 1. Values employed in the sensitivity analysis

\begin{tabular}{llllllllll}
\hline Param.I & $\mathrm{a} 1$ & $\mathrm{R}$ & $\mathrm{a} 2$ & $\mathrm{R}$ & $\mathrm{L}$ & $\mathrm{Q}$ & $\mathrm{D}$ & $\mathrm{k} 1$ & $\mathrm{k} 2$ \\
Series & $10-4 \mathrm{~m} 2 \mathrm{~s}-1$ & $10-2 \mathrm{~m}$ & $10-5 \mathrm{~m} 2 \mathrm{~s}-1$ & $10-2$ a.u. & $10-3 \mathrm{~m}$ & $10-3 \mathrm{~W} / \mathrm{m} 2$ & $10-4 \mathrm{~m}$ & $\mathrm{~W} / \mathrm{mK}$ & $\mathrm{W} / \mathrm{mK}$ \\
\hline 0\% Initial & 1.00 & 2.0 & 2.0 & 1.00 & 3.00 & 1.60 & 1.00 & 250 & 100 \\
$5 \%$ & 1.05 & 2.1 & 2.1 & 1.05 & 3.15 & 1.68 & 1.05 & 262 & 105 \\
$10 \%$ & 1.10 & 2.2 & 2.2 & 1.10 & 3.30 & 1.76 & 1.10 & 275 & 110 \\
$100 \%$ & 2.00 & 4.0 & 4.0 & 2.00 & 6.00 & 3.20 & 2.00 & 500 & 200 \\
\hline
\end{tabular}

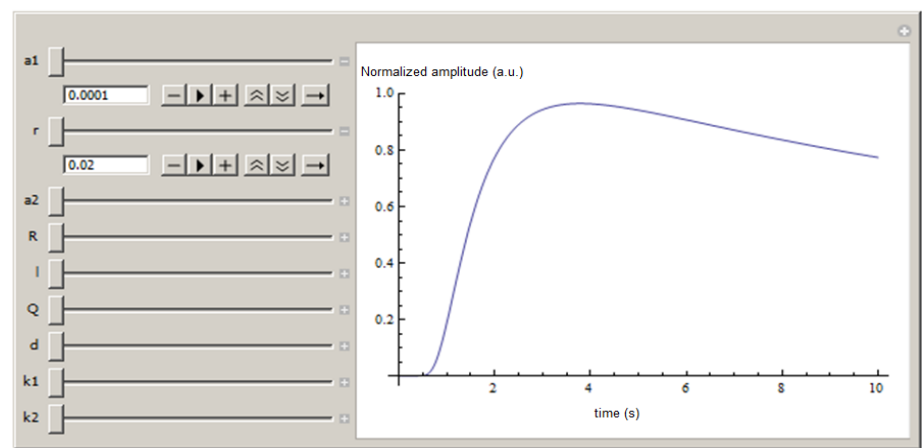

Figure 3. Plot of the temperature corresponding to the initial value of the parameters (with iteration number $\mathrm{m}=2$ ). The changes in temperature can be seen by adjusting the slider of corresponding parameter simulated using Mathematica 


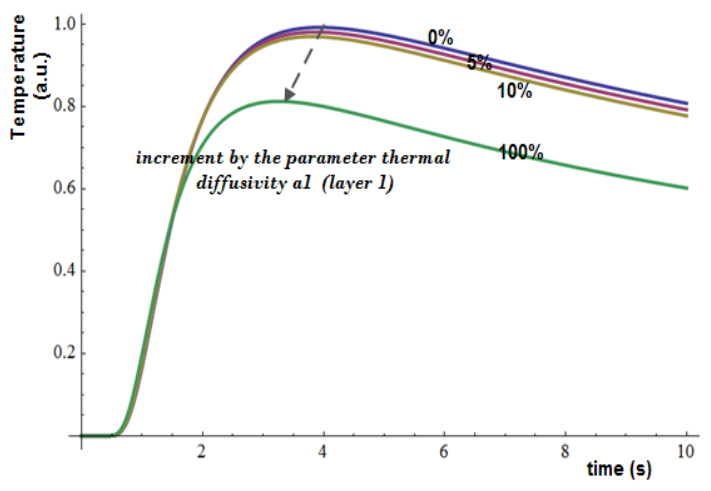

Fig. $4 a$

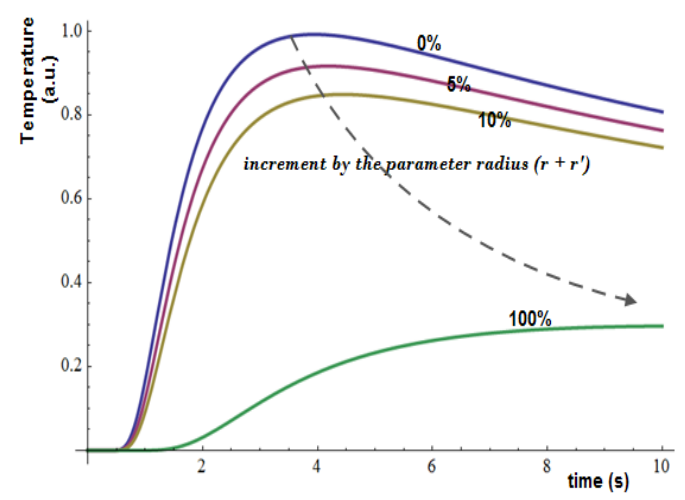

Fig. 4c



Fig. 4 e

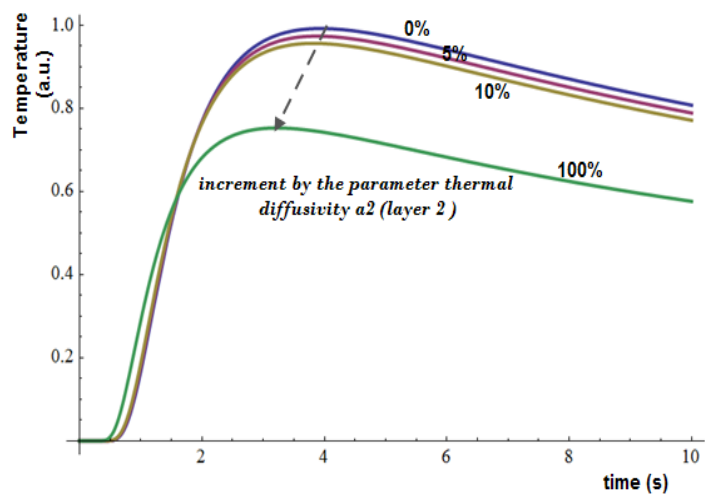

Fig. 4b

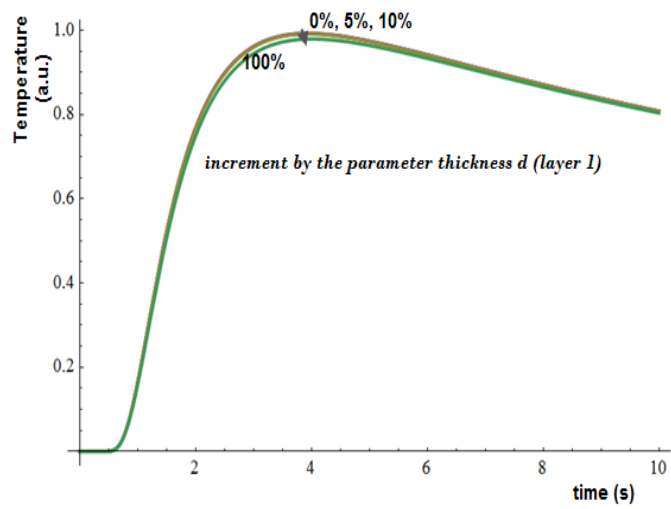

Fig. 4 d

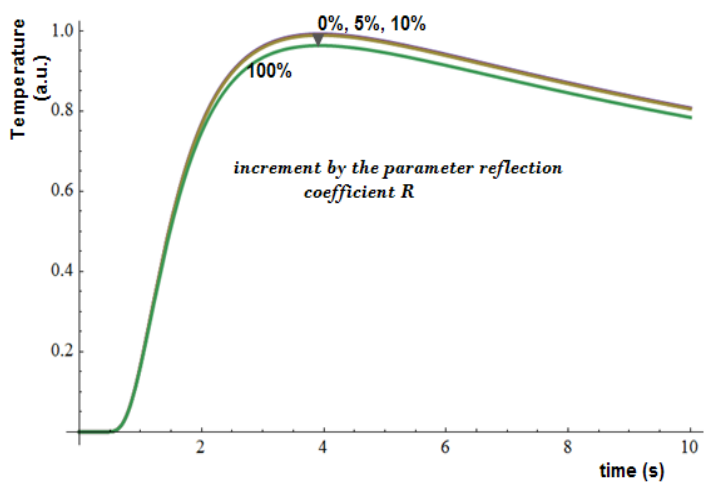

Fig. $4 f$



Fig. 4g

Figure 4. (a), (b), (c), (d), (e), (f) and (g) illustrate the temperature correspond to the 5\%, $10 \%$ and $100 \%$ increment value to the correlated parameters (iteration number $\mathrm{m}=2$ ) 


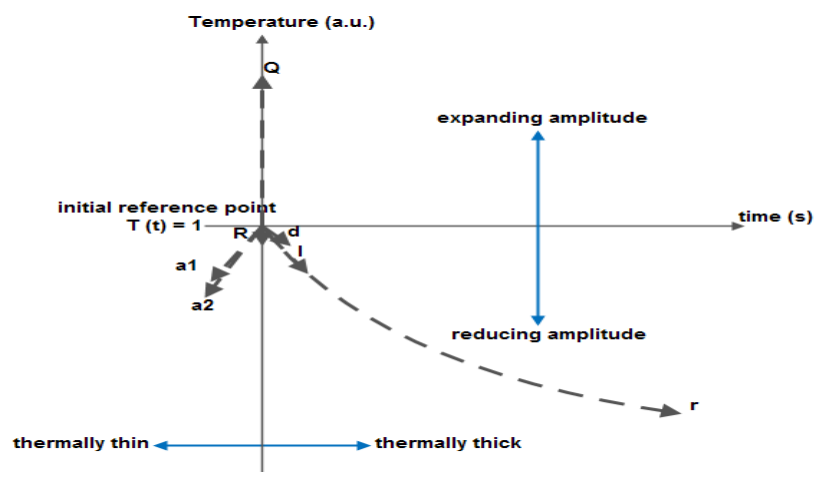

Figure 5. Sensitivity analysis plot which extracted from Fig. 4a, 4b, 4c, 4d, 4e, 4f, and 4g. Parameter k1 and $\mathrm{k} 2$ are insensitive upon the allocated initial values

Figure 5 summarized the results of the sensitivity analysis obtained from the temperature calculation plotted in Figure 4 (a), (b), (c), (d), (e), (f) and (g). It was obviously shown that the ranking of the parameter sensitivity is as the following (from high to low sensitivity order) r, a2, a1, l and R. Q has not been mentioned here because it is only amplitude-dependent parameter which is not involved in the temperature evolution process in the sample. Thermal conductivity of $\mathrm{k} 1$ and $\mathrm{k} 2$ are not sensitive (dummy parameters) as they are a part of the reflection coefficient $R$ which can be significantly sensitive upon the temperature, when $R$ comprising a high ratio approaching to 1 . The effect on parameters $\mathrm{k} 1$ or $\mathrm{k} 2$ can be negligible by selecting very high thermal diffusivity or very low thermal conductivity by the ratio $\frac{\mathrm{k} 1}{\sqrt{\mathrm{a} 1}}=\frac{\mathrm{k} 2}{\sqrt{\mathrm{a} 2}} \rightarrow \infty$, at least $1 \times 10^{6}$ as assigned in Table 1.

We consider first layer become transparent and thermally thin (thickness $\mathrm{d}$ is 100 microns compared to the camera flash duration $\sim 10 \mathrm{~ms}$ ) causing no absorption by the front surface, since the pulse duration was not short enough accordingly high diffusivity materials (such as metal) might be used, thickness of layer 1, d insufficiently sensitive could be explained (where allocated values of first layer's actually imitates metallic foils properties). We found that, parameter $\mathrm{d}$ becomes significantly sensitive when it portion approaching at least 25 times larger compared to the total radius, $r+r^{\prime}$ (Figure 6), which giving $\frac{r \prime}{r}=\frac{1}{25}=0.04$ by taking $r^{\prime}=d$. This ratio $\frac{r \prime}{r}=0.04$ is rather a good factor to keep the size in the semi-infinite configuration, which the existence of the radial thermal wave can be assured. From this result, the thickness of the front layer (layer 1) can be reduced down to the submicron, under the implementation by optimizing the system using shorten the pulse and ring width by pulsed laser and axicon lens [4]. By doing so, made the art of this technique becomes realistic and meaningful. We have made the restriction that the thickness of layer 2 (the substrate) should be respected to the Parker' thickness [1] (within the range $0.5-2 \mathrm{~mm}$ ) in order to confirm the axial thermal wave adequately being generated in a bilayer specimen.

From the discussion above, these reasonable results make sense for the use in the experiment for measuring thermal diffusivity of bilayer specimen. The thickness (d) of layer 1 is rather not sensitive, apparently due to high order thermal diffusivity have been employed for this layer and become thermally thin when $\frac{d}{\mathrm{r}+\mathrm{r}^{\prime}}<25$, which lead thermal wave dominated in radial.

\section{CONCLUSION}

We have conducted a sensitivity analysis of a thin bilayer specimen using simplified converging thermal wave technique. This present work is simple and suitable for ordinary light pulse using camera flash as the optical heating source, and thermocouple as the detector can be alternative to replace the ultra-short pulsed laser and infrared detector. Under this theoretical approach, the simple experimental setup can be implemented and data can be analyzed by fitting the theoretical curve upon the experimental data, in order to obtain unknown parameter such as thermal diffusivity of the particular's layer. A thin layer (layer 1) is significantly sensitive when the portion is at least 25 times larger compared to the total radius, $\mathbf{r}+\mathbf{r}^{\prime}$, and the equivalent ratio $\frac{r \prime}{r}$ ought to be higher or at least 0.04 in order the radial component of converging thermal wave to be efficiently effected. From the results, we recommend the thickness of the front layer (layer 1) can be reduced down to the submicron, under the implementation using ultra-short pulsed laser. 


\section{ACKNOWLEDGEMENTS}

This work is financially supported by Ministry of Higher Education, Putrajaya, Malaysia.

\section{REFERENCES}

[1] W. J. Parker, et al., "Flash Method of determining thermal diffusivity, heat capacity and thermal conductivity," $J$. Appl. Phys., 1961, Vol. 32, pp. 1679-1684.

[2] L. Kehoe, et al., "Application of the laser flash diffusivity method to thin high thermal conductivity materials," Microsystem Technologies, 1998, Vol. 5, No.1, pp.18-21.

[3] P. Cielo, et al., "Thermal diffusivity measurements by the converging-thermal-wave technique," Can. J. Phys., 1986, V1. 64, No. 9, pp. 1172-1177.

[4] F. Murphy, et al., "Development of an algorithm to extract thermal diffusivity for the radial converging wave technique," International Journal of Heat and Mass Transfer, 2005, Vol. 48, No. 7, pp. 1395-402.

[5] Sok Won Kim, et al., "Analysis of thermal diffusivity by parameter estimation in converging thermal-wave technique," International Journal of Heat and Mass Transfer, 2006, Vol. 49, pp. 611-616.

[6] M. S. Husin, et al., "A Simplified Low Cost Converging Thermal Wave Technique for Measuring Thermal Diffusivity of Thin Foils," IOSR Journal of Applied Physics, 2013, pp. 38-46.

[7] Timothy Kehoe, et al., "A Method for Measuring the Thermal Diffusivity of Intermediate Thickness Surface Absorbing Samples and Obtaining the Ratio of Anisotropy by the Converging Wave Flash Method," Int J Thermophys., 2009, Vol. 30, No. 3, pp. 987-1000.

[8] L. D. Favro, et al., "Improving the Resolution of Pulsed Thermal Wave Images with a Simple Inverse Scattering Technique," Journal De Physique IV, Colloque C7, 1994, Vol. 4, pp. 545-550.

[9] D. J. Crowther, et al., "An inverse scattering algorithm applied to infrared thermal wave images," J Appl. Phys. 1993, Vol. 74, pp. 5828-5534. 